\title{
Costa Rica sin Fronteras
}

Pensar en Movimiento:

Revista de Ciencias del Ejercicio y la Salud

ISSN 1659-4436

Vol. 18, No.1, pp. 1- 7

Abre $1^{\circ}$ de enero, cierra 30 de junio, 2020

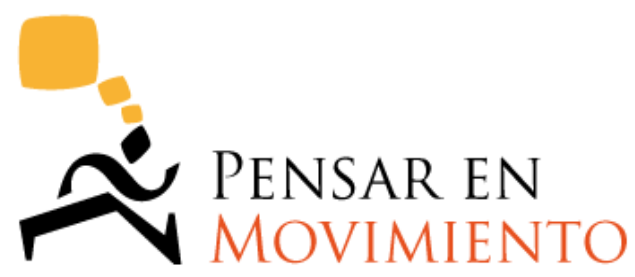

REVISTA DE CIENCIAS DEL EJERCICIO Y LA SALUd

\section{COSTA RICA SIN FRONTERAS \# 21}

En esta sección se publican los resúmenes en español de artículos que han sido publicados por investigadoras/es de universidades costarricenses en otras revistas en el mundo, con su debida referencia al trabajo original, y con una breve explicación de dónde se realizó la investigación. Los artículos originales han sido publicados en otros idiomas; las revistas tienen consejo editorial y manejan un proceso de revisión por pares.

Los resúmenes corresponden a estudios relacionados con las ciencias del ejercicio y la salud, que se conforman a los criterios generales de la revista, esto es, se trata de "... estudios experimentales o que hagan recomendaciones concretas para solucionar problemas o preguntas relevantes (...) trabajos originales o de meta-análisis." Solicitamos a quienes hayan publicado este tipo de trabajos en otros idiomas que no los hagan saber, para incluir sus resúmenes en futuras entregas de esta sección.

\section{Luis Fernando Aragón V., Ph.D., FACSM Director, PENSAR EN MOVIMIENTO}




\section{MAPEO DE LAS ASOCIACIONES ENTRE EL LIPIDOMA PLASMÁTICO, LA RESISTENCIA A LA INSULINA Y LA CURVA DE TOLERANCIA ORAL A LA GLUCOSA}

Beyene, H. B., Hamley, S., Giles, C., Huynh, K., Smith, A., Cinel, M., Mellet, N. A., MoralesScholz, M. G., Kloosterman, D., Howlett, K. F., Kowalski, G. M., Shaw, C. S., Magliano, D. J., Bruce, C. R., Meikle, P. J. (2020). Mapping the Associations of the Plasma Lipidome With Insulin Resistance and Response to an Oral Glucose Tolerance Test (1). The Journal of Clinical Endocrinology and Metabolism, 105(3): e1041-e1055. https://doi.org/10.1210/clinem/dgaa054

Contexto: La resistencia a la insulina sigue siendo un desafío en salud a nivel mundial. Los estudios en lipidómica permiten identificar una variedad de biomarcadores que facilitan una mejor compresión de los mecanismos de resistencia a la insulina relacionados con el metabolismo anormal de los lípidos.

Objetivo: Determinar especies de lípidos en plasma relacionados con índices de resistencia a la insulina y evaluar la respuesta del lipidoma ante una curva de tolerancia a la glucosa.

Diseño experimental y entorno: El presente estudio se realizó en la comunidad y su diseño fue transversal.

Participantes y muestra: Las muestras de plasma (recolectadas a 0 min y 120 min durante la curva de tolerancia a la glucosa) de adultos jóvenes no obesos entre 18 y 34 años ( $n=246)$ se analizaron utilizando espectrometría de masas acoplada a la cromatografía líquida.

Medidas de resultados principales: Las asociaciones entre los índices de resistencia a la insulina y las clases y especies de lípidos (con un término de interacción de sexo), o los cambios en los niveles de lípidos durante la curva de tolerancia a la glucosa, se examinaron utilizando modelos lineares (ajustados a la edad, sexo, índice de masa corporal, colesterol total, HDL y triglicéridos).

Resultados: Algunas especies de lípidos (213 y 199) se asociaron con la evaluación del modelo homeostático de resistencia a la insulina (HOMA) y el área bajo la curva (AUC) de la insulina, respectivamente. Las especies alquilfosfatidilcolina (10), alquenilfostatidilcolina (23) y alquilfosfatidiletanolamina (6) se asociaron con el AUC de la insulina en hombres solamente. Las especies fosfatidilcolina (7) y esfingomielina (5) se asociaron con el AUC de la insulina únicamente en mujeres. Se observó una perturbación en el lipidoma plasmático, particularmente en las especies de acilcarnitina, en respuesta a la curva de tolerancia a la glucosa, y los cambios observados en muchas de las especies de lípidos se asociaron con la AUC de la insulina. 
Conclusiones: El lipidoma plasmático y los cambios en los niveles de lípidos durante una curva de tolerancia a la glucosa se asociaron con los índices de resistencia a la insulina. Estos hallazgos subyacen la participación de especies de lípidos moleculares en la patogénesis de la resistencia a la insulina y posiblemente la diafonía entre la resistencia a la insulina y la regulación específica por sexo del metabolismo de lípidos.

Palabas clave: lipidoma plasmático, resistencia a la insulina, curva de tolerancia a la glucosa, diabetes.

La revista The Journal of Clinical Endocrinology \& Metabolism presenta un Factor de Impacto de 5.605 para el año 2018 según su propia página, así como un índice $\mathrm{H}=328$. Este estudio se realizó en el Instituto de Actividad Física y Nutrición (IPAN: Institute for Physical Activity and Nutrition), Universidad de Deakin, Geelong, Australia (Deakin University, Geelong, Australia). No existe evidencia directa del efecto del ejercicio sobre dichas asociaciones, sin embargo, se sabe que los marcadores utilizados en este estudio, por ejemplo la sensibilidad a la insulina y el perfil lípido, mejoran con la actividad física regular. Por ende, es factible que el ejercicio físico posea un efecto beneficioso sobre las asociaciones descritas. Colaboradora: María Gabriela Morales Scholz. 


\title{
MECANISMOS DE HIPERINSULINEMIA EN JÓVENES NO OBESOS APARENTEMENTE SALUDABLES: EL PAPEL DE LA SECRECIÓN, ACLARAMIENTO Y ACCIÓN DE LA INSULINA Y LAS ASOCIACIONES CON LOS AMINOÁCIDOS PLASMÁTICOS
}

\begin{abstract}
Hamley S, Kloosterman D, Duthie T, Dalla Man C, Visentin R, Mason SA, Ang T, Selathurai A, Kaur G, Morales-Scholz MG, Howlett KF, Kowalski GM, Shaw CS, \& Bruce CR. (2019). Mechanisms of hyperinsulinaemia in apparently healthy non-obese young adults: role of insulin secretion, clearance and action and associations with plasma amino acids. Diabetologia 62: 2310-2324. https://doi.org/10.1007/s00125-019$\underline{04990-y}$
\end{abstract}

Objetivos/hipótesis: El objetivo del presente estudio es examinar la salud metabólica de adultos jóvenes no obesos y aparentemente saludables con el fin de comprender los mecanismos de hiperinsulinemia.

Métodos: Adultos $(\mathrm{N}=254)$ no obesos $(\mathrm{IMC}<30 \mathrm{~kg} / \mathrm{m} 2)$ se sometieron a una curva de tolerancia oral a la glucosa con un radioisótopo estable. La sensibilidad a la insulina, la efectividad de la glucosa y la función de las células beta fueron determinados utilizando modelos mínimos orales. Los individuos fueron estratificados en cuartiles basados en la respuesta de la insulina durante la curva de tolerancia oral a la glucosa, donde el cuartil 1 poseía las respuestas más bajas, mientras que el cuartil 4, las respuestas más altas.

Resultados: Un $13 \%$ de los individuos presentaron una alteración en la glicemia en ayunas $(n=14)$ o intolerancia a la glucosa $(n=19)$, lo que permitió comparar un espectro de respuestas de la insulina, desde normoglicemia a prediabetes. EI IMC ( 24 kg/m2) fue similar entre los cuartiles de insulina, así como en los individuos con alteración en la glicemia e intolerancia a la glucosa. A pesar de presentar excursiones glicémicas similares, los individuos en el cuartil 4 presentaron concentraciones elevadas de insulina, triglicéridos y colesterol. El cuartil 4 presentó la sensibilidad a la insulina más baja acompañada de una secreción a la insulina elevada y un aclaramiento de insulina reducido. Los individuos con alteración en la glicemia en ayunas presentaron una sensibilidad a la insulina y una función de las células beta similar a los individuos en los cuartiles 2 y 3 sin embargo, resultaron más insulino sensibles que los individuos del cuartil 4. Los individuos con intolerancia a la glucosa presentaron un grado semejante de resistencia a la insulina que aquellos del cuartil 4, ellos presentaron un defecto más severo en la función de las células beta. Los aminoácidos ramificados en plasma no se encontraron elevados en los individuos del cuartil 
4, los que presentaron alteración en la glicemia en ayunas o en los individuos con intolerancia a la glucosa.

Conclusiones/interpretación: La hiperinsulinemia en adultos jóvenes, no obesos, normoglicémicos se manifiesta debido a un aumento en la secreción, así como en una reducción en el aclaramiento de la insulina. La caracterización fenotípica individualizada reveló que los individuos más hiperinsulinémicos eran más similares a los individuos con alteración en la glicemia en ayunas que a los individuos con intolerancia a la glucosa. Lo anterior sugiere que los individuos hiperinsulinémicos pueden pertenecer a un continuo hacia la intolerancia a la glucosa. Los aminoácidos ramificados en plasma pueden no ser de utilidad como un biomarcador efectivo para identificar hiperinsulinemia y resistencia a la insulina en adultos jóvenes, no obesos.

Palabras clave: resistencia a la insulina, diabetes, prediabetes, hiperinsulinemia, insulina.

La revista Diabetologia tiene un factor de impacto de 7.113 para 2018. Su ISI Journal Citation Reports Ranking es de 12 entre 145 revistas del área de endocrinología y metabolismo. Este estudio se realizó en el Instituto de Actividad Física y Nutrición (IPAN: Institute for Physical Activity and Nutrition), Universidad de Deakin, Geelong, Australia (Deakin University, Geelong, Australia). No existe evidencia directa del efecto del ejercicio sobre dichas asociaciones, sin embargo, se sabe que los marcadores utilizados en este estudio, por ejemplo la sensibilidad a la insulina y el perfil lípido, mejoran con la actividad física regular. Por ende, es factible que el ejercicio físico posea un efecto beneficioso sobre las asociaciones descritas. Colaboradora: María Gabriela Morales Scholz. 


\title{
EL IMPACTO DEL NIVEL DE ENTRENAMIENTO FÍSICO SOBRE LA ABUNDANCIA ESPECÍFICA DEL TIPO DE FIBRA MUSCULAR DE PROTEÍNAS INVOLUCRADAS EN LA UTILIZACIÓN DE LÍPIDOS INTRAMUSCULARES
}

\author{
Shaw CS, Swinton C, Morales-Scholz MG, McRae N, Erftemeyer T, Aldous A, Murphy RM \\ \& Howlett KF. (2020). Impact of exercise training status on the fiber type-specific \\ abundance of proteins regulating intramuscular lipid metabolism. Journal of Applied \\ Physiology 128(2):379-89. https://doi.org/10.1152/japplphysiol.00797.2019
}

El entrenamiento al ejercicio aumenta la capacidad de oxidación de lípidos durante el ejercicio debido a una mayor utilización de lípidos intramusculares. El presente estudio investigó cuantitativamente el impacto del nivel de entrenamiento físico sobre la abundancia específica del tipo de fibra muscular de proteínas involucradas en la utilización de lípidos intramusculares. Siete individuos entrenados en resistencia aeróbica (V்O2peak $=62.6 \pm$ 4.1 (SD) $\mathrm{mL} \cdot \mathrm{min}-1 \cdot \mathrm{kg}-1$ ) y ocho individuos no entrenados en resistencia aeróbica (V்O2peak $44.9 \pm 5.3 \mathrm{~mL} \cdot \mathrm{min}-1 \cdot \mathrm{kg}-1$ ) completaron una prueba de ejercicio incremental para determinar la oxidación máxima de grasas, así como el consumo máximo de oxígeno. El contenido de lípidos intramusculares específico del tipo de fibra muscular, así como la abundancia de proteínas fueron determinadas por microscopía por inmuno-fluorescencia y por inmuno-blot en fibras musculares únicas agrupadas, así como en tejido muscular entero. Los individuos entrenados en resistencia aeróbica presentaron una mayor tasa de oxidación máxima de grasas $(0.45 \pm 0.15$ vs. $0.19 \pm 0.07 \mathrm{~g} / \mathrm{min}, \mathrm{P}<0.05)$, una mayor proporción de fibras tipo I y un mayor contenido de lípidos intramusculares en comparación con los individuos no entrenados $(P<0.05)$. La abundancia de la lipasa de triglicéridos adiposos, la lipasa sensible a hormonas, las perilipinas 2 y 5 , así como la hidroxicoacilcoenzima A deshidrogenasa fue 2-3 veces más elevada en las fibras tipo I en comparación con las fibras tipo lla $(P<0.05)$. Asimismo, la abundancia de dichas proteínas asociadas a lípidos y de las enzimas oxidativas fue mayor en el músculo entero de los individuos entrenados en resistencia aeróbica. La oxidación máxima de grasas correlacionó con la proporción de fibras tipo I. La abundancia de proteínas involucradas en la regulación del almacenamiento de los lípidos intramusculares y su oxidación es altamente específica del tipo de fibra muscular. La mayor capacidad de oxidación de grasas en los individuos con mayor resistencia aeróbica correspondió a un mayor contenido de lípidos intramusculares y a una elevada abundancia de enzimas lipolíticas y oxidativas en combinación con una mayor proporción de fibras musculares tipo I. 
Palabras clave: ciclismo, ejercicio, metabolismo de grasas, intramuscular, gotas lipídicas, entrenamiento, triacilglicerol.

La revista Journal of Applied Physiology tiene un factor de impacto de 3.140 para 2019, según la página Academic Accelerator. La misma página reporta un índice $H=211$. Este estudio se realizó en el Instituto de Actividad Física y Nutrición (IPAN: Institute for Physical Activity and Nutrition), Universidad de Deakin, Geelong, Australia (Deakin University, Geelong, Australia). Colaboradora: María Gabriela Morales Scholz. 